\title{
Influence of Boundary Conditions and Defects on the Buckling Behavior of SWCNTs via a Structural Mechanics Approach
}

\author{
Vali Parvaneh, ${ }^{1}$ Mahmoud Shariati, ${ }^{2}$ Hamid Torabi, ${ }^{3}$ and Amir Masood Majd Sabeti ${ }^{2}$ \\ ${ }^{1}$ Department of Engineering, Islamic Azad University, Shahrood Branch, 3619943189 Shahrood, Iran \\ ${ }^{2}$ Department of Mechanical Engineering, Shahrood University of Technology, Daneshgah Boulevard, 36199955161 Shahrood, Iran
}

${ }^{3}$ Young Researchers Club, Islamic Azad University, Mashhad Branch, 9186854693 Mashhad, Iran

Correspondence should be addressed to Vali Parvaneh, vali.parvaneh@gmail.com

Received 5 January 2011; Accepted 16 March 2011

Academic Editor: Daniel Lu

Copyright (C) 2011 Vali Parvaneh et al. This is an open access article distributed under the Creative Commons Attribution License, which permits unrestricted use, distribution, and reproduction in any medium, provided the original work is properly cited.

\begin{abstract}
The effects of boundary conditions and defects on the buckling behavior of SWCNTs are investigated using a structural mechanics model. Due to the application of carbon nanotubes in different fields such as NEMS, where they are subjected to different loading and boundary conditions, an investigation of buckling behavior of nanotubes with different boundary conditions is necessary. Critical buckling loads and the effects of vacancy and Stone-Wales defects were studied for zigzag and armchair nanotubes with various boundary conditions and aspect ratios (length/diameter). The comparison of our results with those of the buckling of shells with cutouts indicates that vacancy defects in carbon nanotubes can most likely be modeled as cutouts of the shells. Finally, a hybrid vacancy defect and Stone-Wales defect are also developed, and their effect on the critical buckling loads is studied.
\end{abstract}

\section{Introduction}

In 1991, Iijima [1] discovered carbon nanotubes, which became the focus of many researchers. Due to the unique properties of this phenomenal material in different domains, many devices use carbon nanotubes for optimum function. Therefore, the mechanical analysis of nanotubes must be done for different boundary and loading conditions. Many researchers have turned finite element and continuum methods. Odegard et al. [2] developed an equivalent-continuum model to obtain the geometrical and mechanical properties of truss links by equalizing the molecular potential energies and mechanical strain energy of a representative continuum truss model. Various finite element methods have been applied for prediction of mechanical properties of carbon nanotubes. One of them is a structural mechanics approach developed by $\mathrm{Li}$ and Chou [3]. This model considers a single-walled carbon nanotube as a geometrical frame-like structure so that primary bonds between atoms act as loadbearing beam members, while the carbon atoms are seen as joints of these members. By linking structural mechanics and molecular mechanics, they have obtained the sectional property parameters of these beam members. Cao and
Chen [4] investigated the effects of chirality and boundary conditions on the elastic properties and buckling behavior of single-walled carbon nanotubes using atomistic simulations. They also calculated the critical buckling strains for different lengths and diameter. Their recent atomistic simulation showed that the axial compressive buckling behaviors are highly sensitive to the displacement increment used in the numerical analysis, which may have contributed to the contradictory results in the literature when different displacement increments were used. They have also proposed a new targeted molecular mechanics method to remove the effect of displacement increment so as to achieve a better understanding of the effects of tube length and diameter on SWCNT buckling.

Due to the application of carbon nanotubes in various industries such as NEMS, mechanical analysis of these nanotubes under different loading and boundary conditions is necessary. Amongst these analyses, axial buckling is very important. Thus, we have tried to predict the critical buckling loads of single-walled carbon nanotubes. The method employed for this analysis is a structural mechanics approach. Different defects may exists in carbon nanotubes, which have an effect on the critical buckling loads. Therefore, 


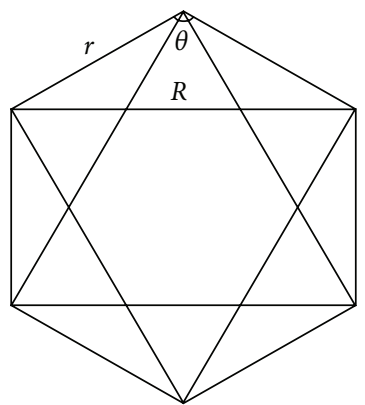

(a)

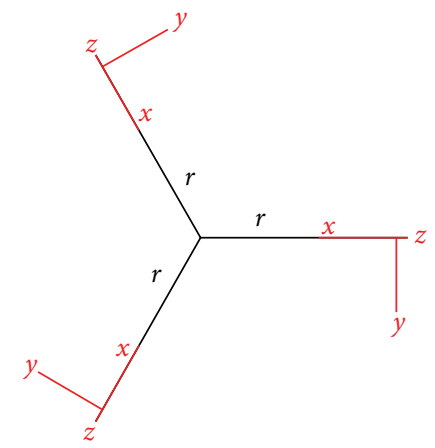

(b)
Figure 1: (a) A hexagonal unit cell, and (b) location of local coordinates of each connector.

the influence of various vacancy and Stone-Wales defects is also investigated in the analysis.

\section{Simulation Method}

2.1. Application of the Structural Model to SWCNTs. We have proposed a structural mechanics method to model the carbon nanotubes. The detailed derivation procedure for the formulation and other features of this model can be found in our previous work [5].

The total steric potential energy due to interactions between carbon atoms can be represented by [5]

$$
u_{\text {total }}=u_{r}+u_{\theta}+u_{\phi}+u_{\omega}
$$

where $u_{r}, u_{\theta}, u_{\phi}$, and $u_{\omega}$ are bond energies associated with bond stretching, angle variation or bond bending, dihedral angle torsion, and out-of-plane torsion, respectively.

In this model, Morse potentials are employed for stretching and bending potentials, and a periodic type of bond torsion is applied for torsion and out-of-plane torsion interactions

$$
\begin{gathered}
u_{r}=D_{e}\left\{\left[1-e^{-\beta\left(r-r_{0}\right)}\right]^{2}-1\right\}, \\
u_{\theta}=\frac{1}{2} k_{\theta}\left(\theta-\theta_{0}\right)^{2}\left[1+k_{\text {sextic }}\left(\theta-\theta_{0}\right)^{4}\right], \\
u_{\phi}=\frac{1}{2} k_{\phi}\left[1+\cos \left(n \phi-\phi_{0}\right)\right], \\
u_{\omega}=\frac{1}{2} k_{\omega}\left[1+\cos \left(n \omega-\omega_{0}\right)\right] .
\end{gathered}
$$

As indicated in Figures 1(a) and 2, a nonlinear axial spring is used for modeling of the angle variation interaction between atoms. The relationship between changes in the bond and the corresponding change in length of the spring for small displacements can be expressed simply by (7) [6]

$$
\Delta \theta \approx \frac{2(\Delta R)}{r_{0}}, \quad r_{0}=0.142 \mathrm{~nm}
$$

Therefore, we can simplify (3) to (7).

$$
u_{\theta}=\frac{2}{r_{0}^{2}} k_{\theta}\left(R-R_{0}\right)^{2}\left[1+\frac{16}{r_{0}^{4}} k_{\text {sextic }}\left(R-R_{0}\right)^{4}\right] \text {. }
$$

The stretch force, the angle variation moment, the dihedral angle torque, and out-of-plane torque can be obtained from differentiations of (2), (7), (4), and (5) as functions of bond stretch, bond angle, dihedral angle, and out-of-plane angle variation, respectively:

$$
\begin{aligned}
& F\left(r-r_{0}\right) \\
& =2 \beta D_{e}\left[1-e^{-\beta\left(r-r_{0}\right)}\right] e^{-\beta\left(r-r_{0}\right)}, \\
& F\left(R-R_{0}\right) \\
& =\frac{4}{r_{0}^{2}} k_{\theta}\left(R-R_{0}\right)\left[1+\frac{16}{r_{0}^{4}}\left(1+\frac{4}{r_{0}^{2}}\right) k_{\text {sextic }}\left(R-R_{0}\right)^{4}\right], \\
& T\left(\phi-\phi_{0}\right) \\
& =\frac{1}{2} k_{\phi} n \sin \left(n \phi-\phi_{0}\right), \\
& T\left(\omega-\omega_{0}\right) \\
& =\frac{1}{2} k_{\omega} n \sin \left(n \omega-\omega_{0}\right) .
\end{aligned}
$$

A nonlinear connector is considered for modeling of the stretching and torsional interactions and a nonlinear spring for modeling of the angle variation interaction (see Figure 2). Carbon atoms in ABAQUS are modeled by a discrete rigid sphere so that connector elements between toms are adjoined to reference points at the center of the sphere and a local coordinate is set at the center of each atom (see Figures 1(b) and 2). This local coordinate is a combination of a Cartesian coordinate for stretching and a rotational coordinate for torsion. The $X$-direction of these coordinates is in the connector direction, and the $Z$-direction is vertical to the central axis of the nanotube. Because we can only use a linear spring in the CAE space of ABAQUS, by changing the linear spring command to a nonlinear spring command in the input file, and by applying the nonlinear data for $F(\Delta R)$ versus $\Delta R$ using (9), we can apply the bond bending spring to the model. For applying bond stretch and torsion forces to the connectors, we can apply the nonlinear stiffnesses in three directions $(X, Y, Z)$ directly. For stretching stiffness in the $X$-direction, we can obtain the nonlinear data for $F(\Delta r)$ versus $\Delta r$ by (8), and for torsional stiffness in $X$-direction, we can obtain the nonlinear data for $T(\Delta \phi)$ versus $\Delta \phi$ by (10). For torsional stiffness in the Y direction, we can obtain the nonlinear data for $T(\Delta \omega)$ versus $\Delta \omega$ by $(11)$.

Here, we take $E=1170 \mathrm{GPa}$ and $v=0.196$ for the Young's modulus and Poisson's ratio of single-walled carbon nanotubes, respectively. As shown in our previous work, we used $k_{r}=800 \mathrm{nN} / \mathrm{nm}, k_{\theta}=1.42 \mathrm{nN} / \mathrm{nm} \cdot \operatorname{Rad}^{-2}, k_{\phi}=k_{\omega}=$ $0.0418 \mathrm{nN} . \mathrm{nm}$, which are consistent with the values reported in the literature. This structural model was successfully used for predicting the mechanical properties and axial buckling behavior of single-walled carbon nanotubes. It is employed here for the bending buckling of single-walled carbon nanotubes. 

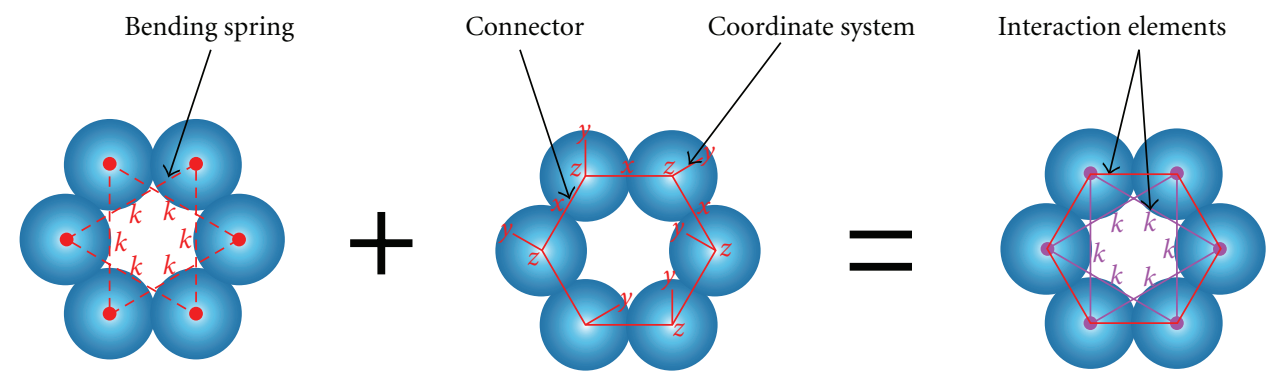

FIGURE 2: Spring and connector elements corresponding to the interactions of carbon atoms. (a) The angle variation interactions, (b) the stretching and torsional interactions, and (c) total interactions [5].

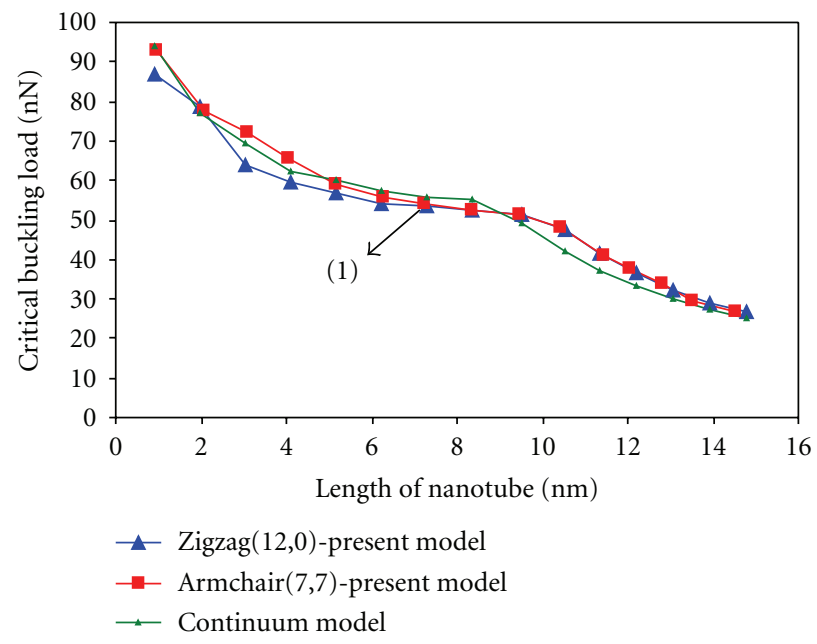

(a)

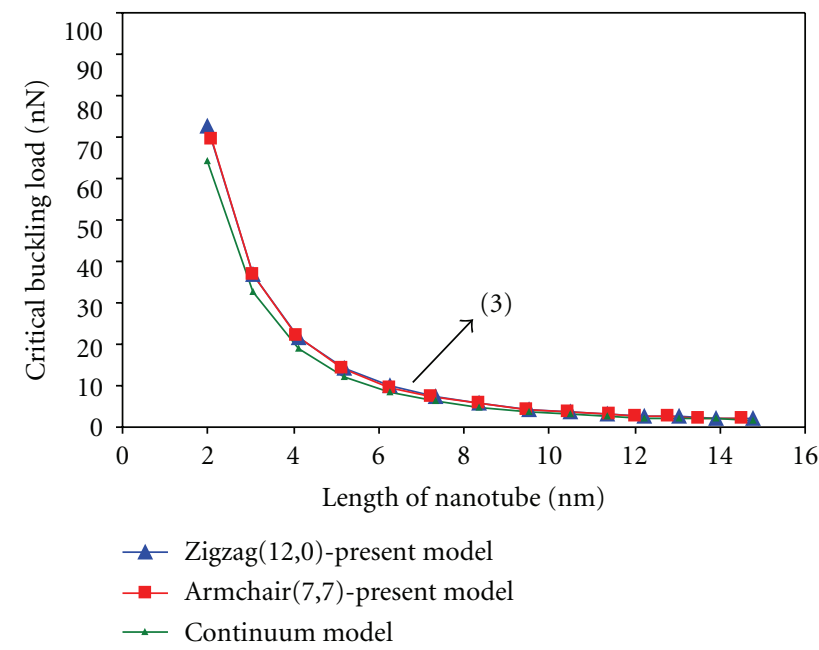

(c)

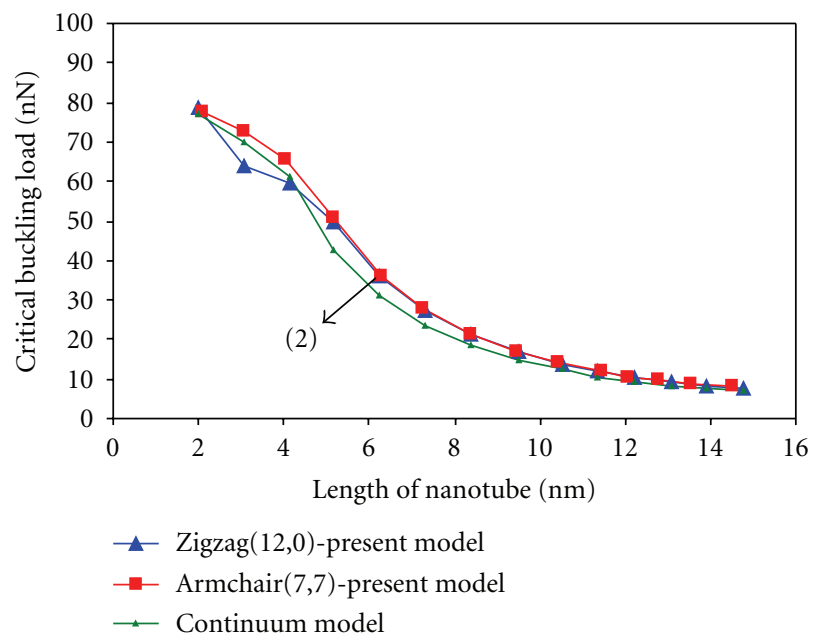

(b)

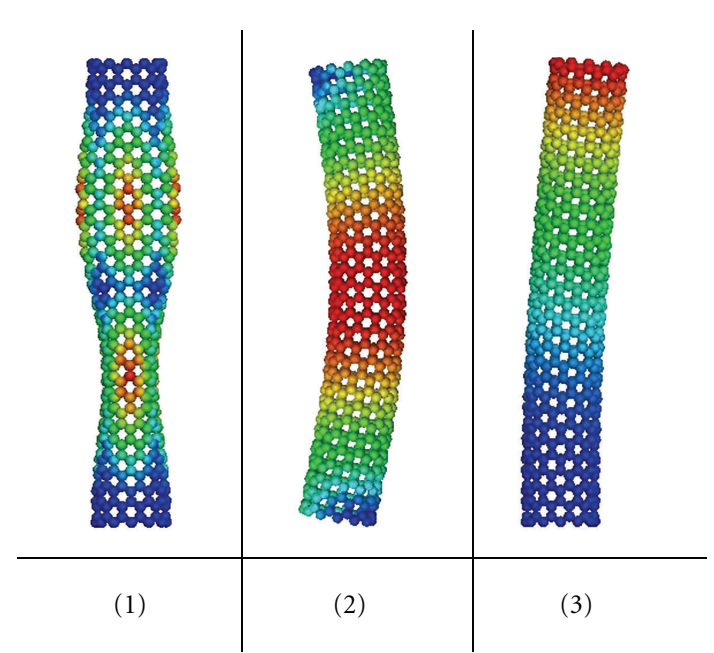

(d)

FIGURE 3: Comparison the present results to results of the continuum model with the critical buckling load curves for (a) fixed ends, (b) simply supported, and (c) free end boundary conditions.

\section{Results and Discussion}

3.1. Buckling of Perfect Single-Walled Carbon Nanotubes. The critical buckling loads are predicted by a new structural model. Zigzag $(12,0)$ and armchair $(7,7)$ SWNTs with various lengths were employed for this study. Figure 3 shows the critical buckling loads for different aspect ratios $(L / d)$ of nanotubes with different boundary conditions ( (a) fixed ends, (b) simply supported, and (c) free end). The results for fixed ends boundary conditions were shown in our previous 


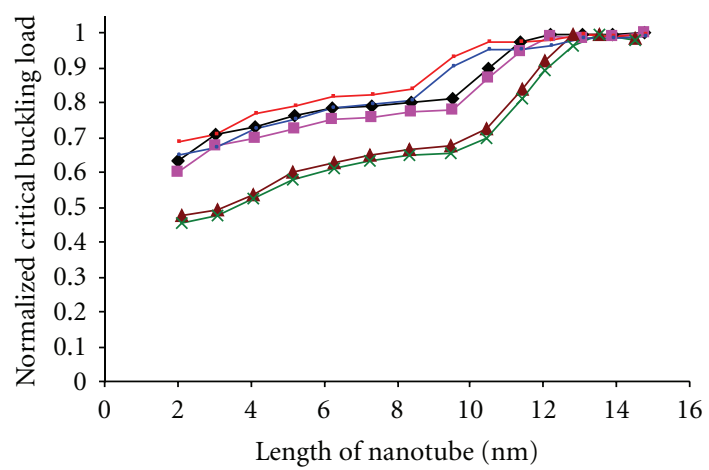

(a)
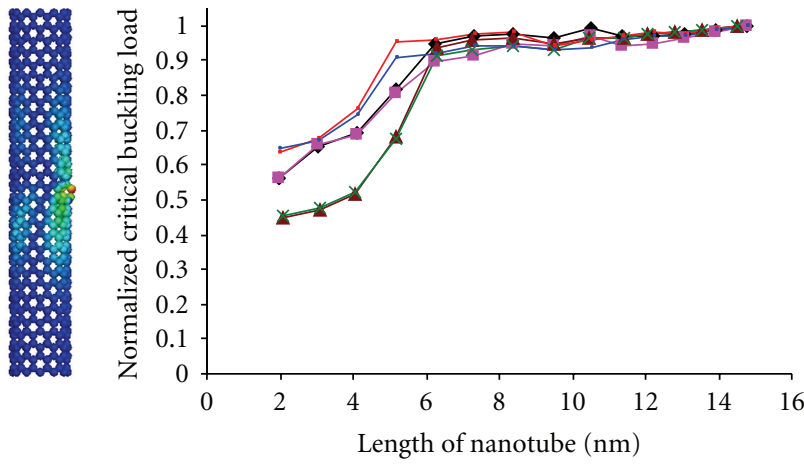

(b)

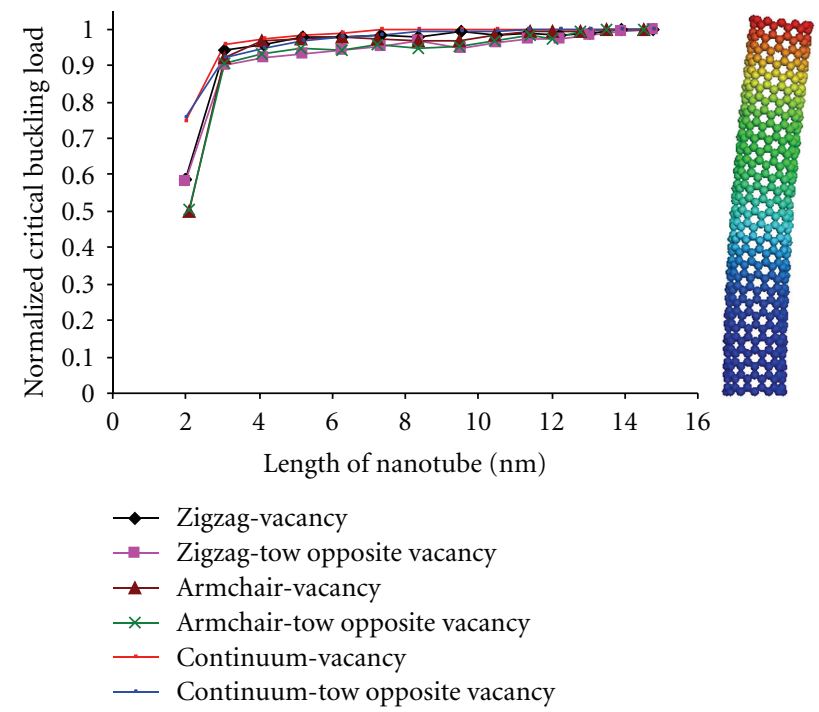

(c)

Figure 4: Normalized critical buckling load curves and comparison between mode shapes of buckling of present model and those from continuum model for (a) fixed ends, (b) simply supported, and (c) free end boundary conditions.

work. However, the curve for the critical buckling loads versus aspect ratios of nanotubes for fixed ends boundary conditions is exhibited just for comparing in this paper.

With increasing nanotube length, shell and then Euler modes will occur. When the Euler buckling mode appears, the critical buckling load follows from the Euler equation. Therefore, we can calculate the critical buckling loads by Euler equations easily for sufficiently long carbon nanotubes. It should be noted that with decreasing bonds on the ends of nanotubes, shell mode shapes will occur at shorter lengths. Therefore, we can also use the Euler equation for shorter nanotubes. Meanwhile, the results of the present work are compared with those from a simple continuum mechanics model in ABAQUS.

The mode shapes according to displacement counters also are represented in Figure 3 for (a) fixed ends, (b) simply supported, and (c) free end boundary conditions, respectively. For simply supported boundary condition, the nanotube ends are pinned and can rotate about the axis lies in the planes at the ends of the nanotube. As shown in Figure 3, for nanotubes with the same length, the mode shapes are different. With decreasing the degrees of freedom in boundary conditions, carbon atoms will move less freely. Therefore, this will lead to the local buckling (shell buckling mode) of CNTs and postpone the global buckling (Euler buckling mode). This is the reason of increasing in the critical buckling load for fixed-fixed boundary condition in comparison to other boundary conditions. Therefore, the mode shapes and the critical buckling loads depend on the boundary conditions as well as the nanotube length. When the shell mode occurs, the boundary conditions do not affect the critical buckling loads.

With an effective wall thickness of $0.34 \mathrm{~nm}$ for SWCNTs, we cannot model them with continuum shell models because the Euler mode will occur too early. An equivalent wall thickness for the continuum shell model that predicts the critical buckling load and the corresponding mode shapes correctly are needed. In Figure 3, we have compared the present results to those of the simple continuum model. The value of the effective thickness for the nanotube is $0.066 \mathrm{~nm}$ [7]. In conclusion, it can be seen that the results of this continuum model are in acceptable agreement with the present model. When the shell buckling mode occurs, the critical buckling loads from continuum model are more than those from 


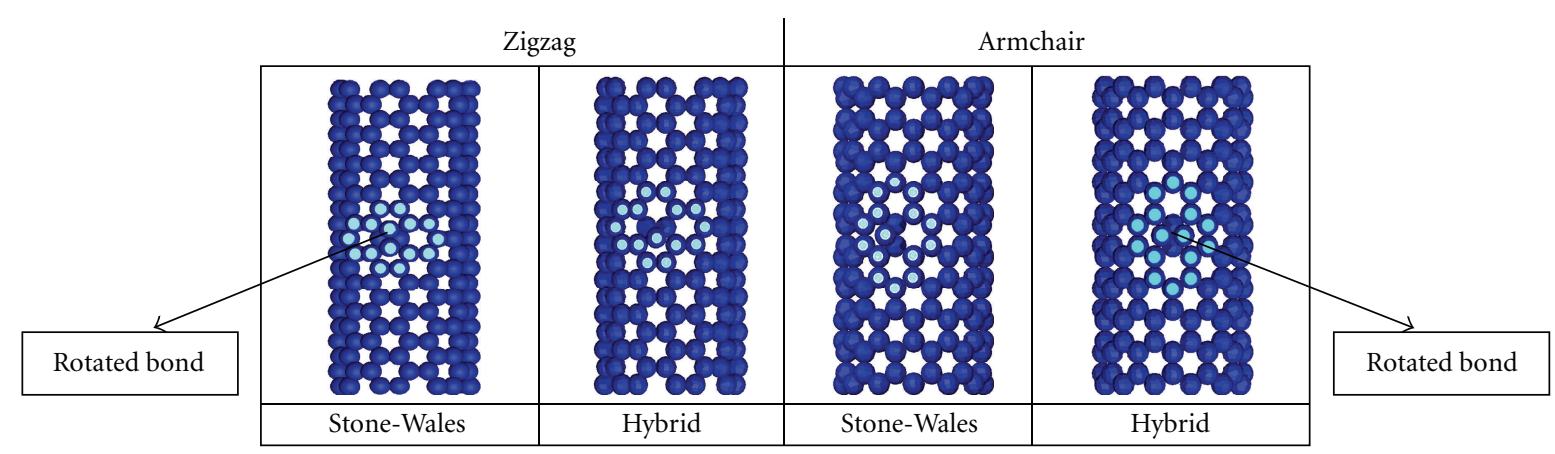

FIgURE 5: Critical buckling loads of defective nanotubes with various aspect ratios for (a) Zigzag and (b) Armchair.

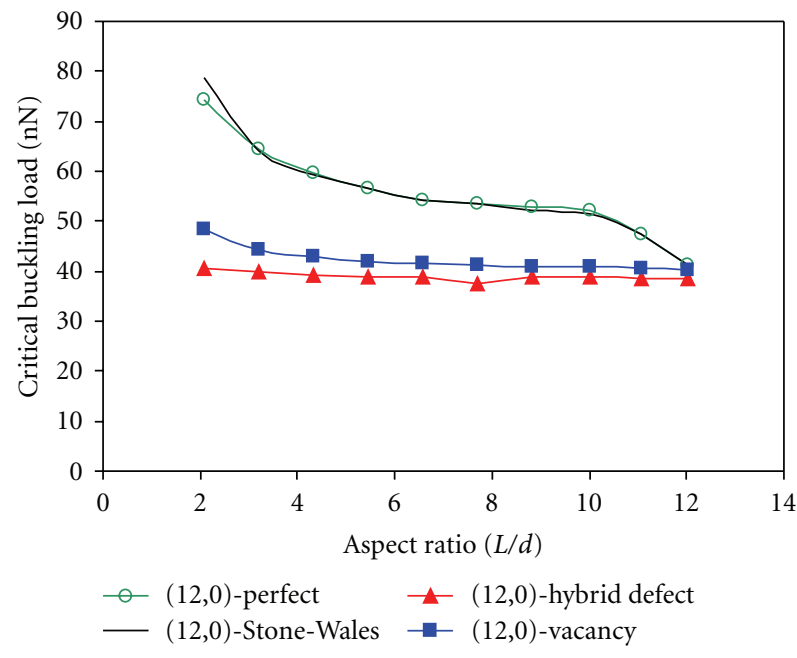

(a)

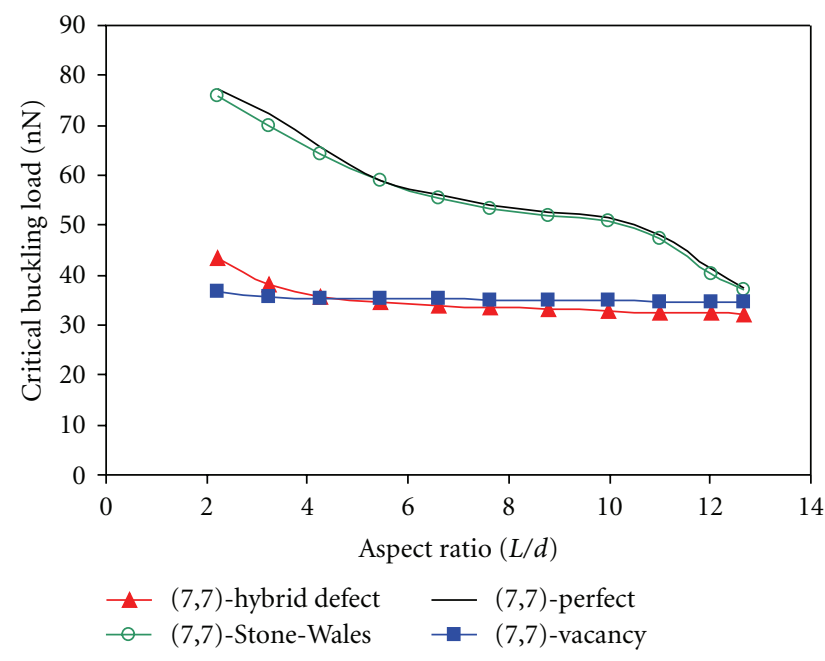

(b)

FIgURE 6: Scheme view of hybrid and Stone-Wales defects in zigzag and armchair nanotubes.

present model, but when the Euler buckling mode occurs, the critical buckling loads from the continuum model are lower than those from the present model. This point shows that the adopted thickness for the continuum model is ideal.

\subsection{Buckling of Defective Single-Walled Carbon Nanotubes.} Vacancies result from missing carbon atoms in the CNT walls. The defects included are single vacancies (one atom missing) and two opposite single vacancies (two atoms missing). By removing the carbons atoms from nanotube, all of interactions (springs and connectors) between bonds will remove. All of the defects are situated in the middle of the nanotube.

Normalized critical buckling load graphs for various lengths of zigzag and armchair nanotubes and boundary conditions are shown in Figure 4. Nanotubes include single vacancy defects at the middle of the nanotube. As indicated in Figure 4, the vacancy defects have a very weak effect on the critical buckling loads when the Euler mode occurs.

Defects are generated in the synthesizing process, and they can also be caused by mechanical manipulation. The most typical structural defects are Stone-Wales defects [8].
Stone-Wales defects in carbon nanotubes are generated under the certain conditions. These topological defects are regions in a crystal where the normal chemical bonding environment is topologically different from the surroundings. The carbon nanotubes contain regions where the number of atoms in a ring is different from six, while the total number of atoms remains the same. Stone and Wales showed that a dipole consisting of a pair of 5-7 rings can be created by rotating the $\mathrm{C}-\mathrm{C}$ bond in a hexagonal network by $90^{\circ}$. As shown in Figure 5, in this rotation, four hexagons are changed into two heptagons and two pentagons.

Unlike the vacancy defects in which bonds have been removed, the carbon bonds in Stone-Wales defects will not remove but will rotate. This defect is in the middle of the nanotube.

The critical buckling loads were obtained for nanotubes including a Stone-Wales defect and two opposite StoneWales defects with fixed ends boundary conditions (see Figure 6).

Stone-Wales defects do not have a significant effect on the critical buckling loads, and even two opposite StoneWales defects barely decrease the critical buckling loads. 


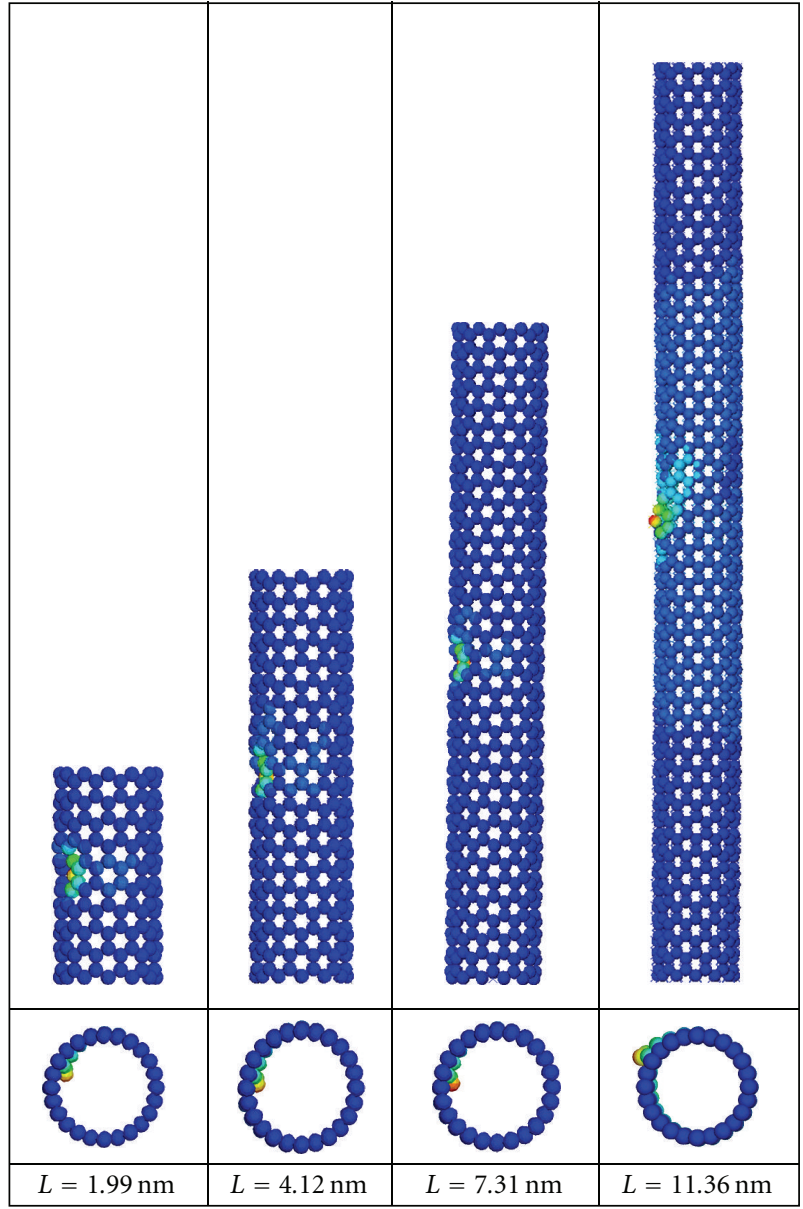

Figure 7: Influence of the hybrid defect on the mode shape of a zigzag $(12,0)$ nanotube for various lengths.

As mentioned above, Euler modes will occur at shorter lengths when bonds decrease and the effect of defects on the critical buckling loads also therefore decreases. Therefore, Stone-Wales defects do not affect the critical buckling loads under the other boundary conditions in general.

The mode shapes of nanotubes with Stone-Wales defects are same as those of perfect nanotubes. At the length of $1.988 \mathrm{~nm}$, Stone-Wales defects do change the mode shape, however.

3.3. Compound of Vacancy and Stone-Wales Defect. A hybrid vacancy defect and Stone-Wales defect was developed, and its effect on the critical buckling loads was studied. Vacancies result from missing carbon atoms in the CNT walls. This can happen when CNTs are subjected to irradiation. When missing carbon atoms occur exactly on the Stone-Wales defect, a hybrid defect may be created in the nanotube. The configuration of this hybrid defect is shown in Figure 5. In this defect, one carbon atom is missing from atoms included in a Stone-Wales defect. The effect of this defect on the critical bucking loads was investigated and compared with those of single vacancy defects (see Figure 6). It can be seen that the hybrid defect decreases the critical buckling loads more than single vacancy defects do, though two carbon atoms are missing in both of them. In addition, the mode shapes of defective nanotubes with a hybrid defect at the middle of the nanotubes are illustrated in Figure 7. The mode shapes due to the hybrid defect and vacancy defect are approximately the same.

\section{Conclusion}

The effects of vacancy and Stone-Wales defects as well as boundary conditions on the axial buckling behavior of carbon nanotubes were studied via a structural model. We proposed a hybrid vacancy/Stone-Wales defect, and its effect on the critical buckling loads was investigated. From these investigations, the following results can be concluded.

(1) An effective thickness of $0.066 \mathrm{~nm}$ is suitable for perfect and defective nanotubes with a diameter of $0.95 \mathrm{~nm}$ and different boundary conditions.

(2) Stone-Wales defects have a weak effect on the critical buckling loads under the critical boundary condition of fixed ends.

(3) With decreasing bonds from the boundaries of nanotubes, the Euler mode occurs earlier, and the effect of vacancy defects decreases.

(4) Hybrid defects decrease the critical buckling loads more than single vacancy defects.

\section{References}

[1] S. Iijima, "Helical microtubules of graphitic carbon," Nature, vol. 354, no. 6348, pp. 56-58, 1991.

[2] G. M. Odegard, T. S. Gates, L. M. Nicholson, and K. E. Wise, "Equivalent-continuum modeling of nano-structured materials," Composites Science and Technology, vol. 62, no. 14, pp. 1869-1880, 2002.

[3] C. Li and T. W. Chou, "A structural mechanics approach for the analysis of carbon nanotubes," International Journal of Solids and Structures, vol. 40, no. 10, pp. 2487-2499, 2003.

[4] G. Cao and XI. Chen, "The effects of chirality and boundary conditions on the mechanical properties of single-walled carbon nanotubes," International Journal of Solids and Structures, vol. 44, no. 17, pp. 5447-5465, 2007.

[5] V. Parvaneh, M. Shariati, and A. M. Majd Sabeti, "Investigation of vacancy defects effects on the buckling behavior of SWCNTs via a structural mechanics approach," European Journal of Mechanics, A/Solids, vol. 28, no. 6, pp. 1072-1078, 2009.

[6] G. M. Odegard, T. S. Gates, L. M. Nicholson, and K. E. Wise, "Equivalent-continuum modeling with application to carbon nanotubes," NASA/TM, 211454, 2002.

[7] B. I. Yakobson, C. J. Brabec, and J. Bernholc, "Nanomechanics of carbon tubes: instability beyond linear response," Physical Review Letters, vol. 76, pp. 2511-2514, 1996.

[8] A. J. Stone and D. J. Wales, "Theoretical studies of icosahedral C and some related species," Chemical Physics Letters, vol. 128, no. 5-6, pp. 501-503, 1986. 

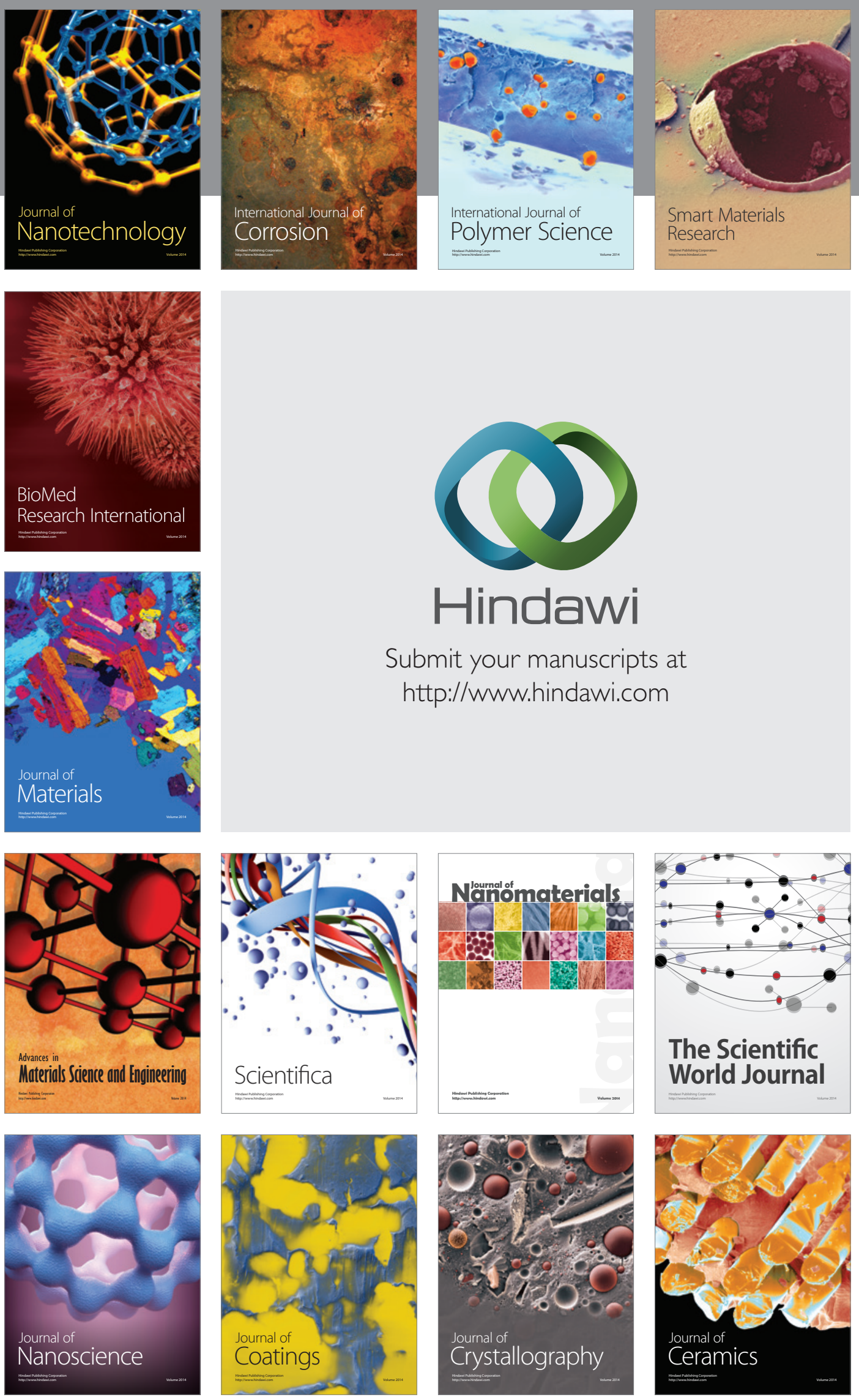

The Scientific World Journal

Submit your manuscripts at

http://www.hindawi.com

\section{World Journal}

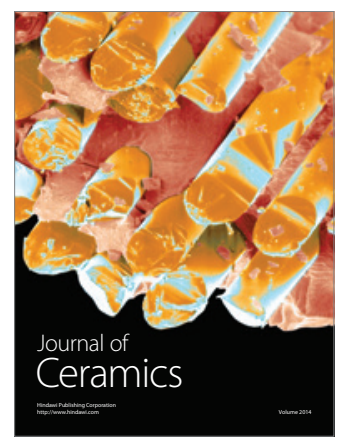

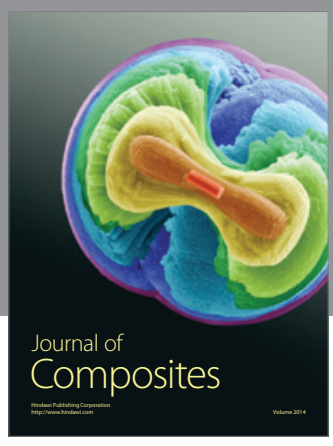
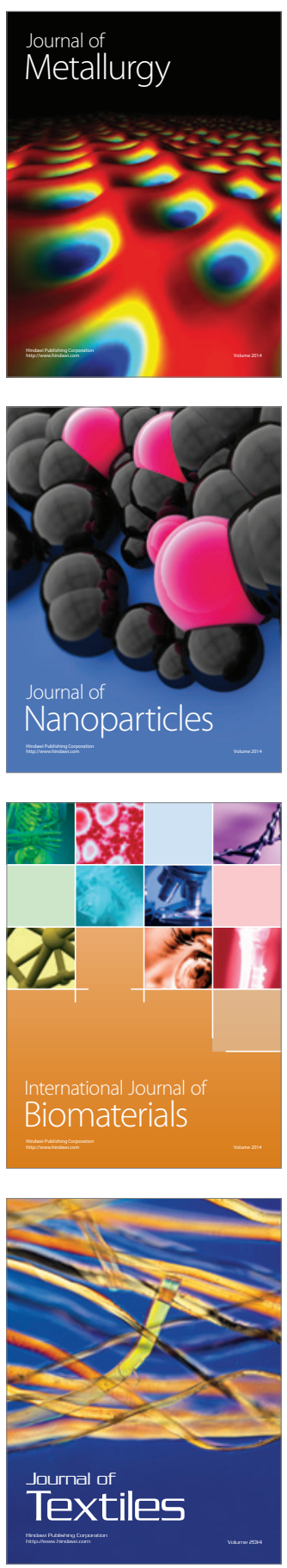